\title{
Efficacy of single-hormone and dual-hormone artificial pancreas during continuous and interval exercise in adult patients with type 1 diabetes: randomised controlled crossover trial
}

\author{
Nadine Taleb $^{1,2} \cdot$ Ali Emami ${ }^{1,3} \cdot$ Corinne Suppere $^{1} \cdot$ Virginie Messier $^{1} \cdot$ \\ Laurent Legault $^{4}$ - Martin Ladouceur ${ }^{5}$ - Jean-Louis Chiasson ${ }^{5,6}$ - Ahmad Haidar ${ }^{7,8}$. \\ Rémi Rabasa-Lhoret ${ }^{1,6,9}$
}

Received: 25 May 2016 / Accepted: 16 August 2016/Published online: 4 October 2016

(C) Springer-Verlag Berlin Heidelberg 2016

\begin{abstract}
Aims/hypothesis The aim of this study was to assess whether the dual-hormone (insulin and glucagon) artificial pancreas reduces hypoglycaemia compared with the single-hormone (insulin alone) artificial pancreas during two types of exercise. Methods An open-label randomised crossover study comparing both systems in 17 adults with type 1 diabetes (age, $37.2 \pm 13.6$ years; $\mathrm{HbA}_{1 \mathrm{c},} 8.0 \pm 1.0 \%$ [63.9 $\left.\pm 10.2 \mathrm{mmol} / \mathrm{mol}\right]$ ) during two exercise types on an ergocycle and matched for energy expenditure: continuous $\left(60 \% \dot{V} \mathrm{O}_{2 \text { peak }}\right.$ for $\left.60 \mathrm{~min}\right)$ and interval $(2 \mathrm{~min}$ alternating periods at $85 \%$ and $50 \%$ $\dot{V} \mathrm{O}_{2 \text { peak }}$ for $40 \mathrm{~min}$, with two 10 min periods at $45 \% \dot{V} \mathrm{O}_{2 \text { peak }}$ at the start and end of the session). Blocked randomisation (size of four) with a 1:1:1:1 allocation ratio was computer generated. The artificial pancreas was applied from 15:30 hours until 19:30 hours; exercise was started at 18:00 hours and announced
\end{abstract}

20 min earlier to the systems. The study was conducted at the Institut de recherches cliniques de Montréal.

Results During single-hormone control compared with dualhormone control, exercise-induced hypoglycaemia (plasma glucose $<3.3 \mathrm{mmol} / 1$ with symptoms or $<3.0 \mathrm{mmol} / 1$ regardless of symptoms) was observed in four (23.5\%) vs two $(11.8 \%)$ interventions $(p=0.5)$ for continuous exercise and in six (40\%) vs one (6.25\%) intervention $(p=0.07)$ for interval exercise. For the pooled analysis (single vs dual hormone), the median (interquartile range) percentage time spent at glucose levels below $4.0 \mathrm{mmol} / \mathrm{l}$ was $11 \%(0.0-46.7 \%)$ vs $0 \%(0-0 \%$; $p=0.0001)$ and at glucose levels between 4.0 and $10.0 \mathrm{mmol} / \mathrm{l}$ was $71.4 \%(53.2-100 \%)$ vs $100 \%(100-100 \% ; p=0.003)$. Higher doses of glucagon were needed during continuous $(0.126 \pm 0.057 \mathrm{mg})$ than during interval exercise $(0.093$ $\pm 0.068 \mathrm{mg})(p=0.03)$, with no reported side-effects in all interventions.
A. Haidar and R. Rabasa-Lhoret are joint senior authors.

Electronic supplementary material The online version of this article (doi:10.1007/s00125-016-4107-0) contains peer-reviewed but unedited supplementary material, which is available to authorised users.

Rémi Rabasa-Lhoret

remi.rabasa-1horet@ircm.qc.ca

1 Institut de recherches cliniques de Montréal, 110 Avenue des Pins Ouest, Montréal, Québec, Canada H2W 1R7

2 Division of Biomedical Sciences, Faculty of Medicine, Université de Montréal, Montréal, Québec, Canada

3 Division of Experimental Medicine, Department of Medicine, McGill University, Montréal, Québec, Canada

4 Montreal Children's Hospital, McGill University Health Centre, Montréal, Québec, Canada
5 Centre de recherche du Centre hospitalier de l'université de Montréal (CRCHUM), Montréal, Québec, Canada

6 Montreal Diabetes Research Center, Montréal, Québec, Canada

7 Department of Biomedical Engineering, Faculty of Medicine, McGill University, Montréal, Québec, Canada

8 Division of Endocrinology, Faculty of Medicine, McGill University, Montréal, Québec, Canada

9 Nutrition department, Faculty of Medicine, Université de Montréal, Montréal, Québec, Canada 
Conclusions/interpretation The dual-hormone artificial pancreas outperformed the single-hormone artificial pancreas in regulating glucose levels during announced exercise in adults with type 1 diabetes.

Trial registration: ClinicalTrials.gov NCT01930110

Funding: Société Francophone du Diabète and Diabète Québec

Keywords Artificial pancreas · Exercise - Glucagon · Hypoglycaemia $\cdot$ Type 1 diabetes

\begin{abstract}
Abbreviations
IQR Interquartile range

MARD Mean absolute relative difference
\end{abstract}

\section{Introduction}

In addition to insulin replacement therapy, patients with type 1 diabetes should maintain a healthy lifestyle that includes regular physical activity [1]. Exercise is associated with several health benefits for patients with type 1 diabetes such as improved physical fitness, reduced cardiovascular risk factors and reduced insulin requirements $[2,3]$. Nevertheless, the majority of patients with type 1 diabetes do not meet the recommended physical activity levels mostly because of fear of exerciseinduced hypoglycaemia $[4,5]$. The inability to decrease plasma insulin levels when the glucose utilisation rate and insulin sensitivity are increased by exercise is the main factor that sets the stage for hypoglycaemia [6]. Another contributory factor in this setting is the deficiency of counter-regulatory hormones, mainly glucagon, cortisol and catecholamines [6, 7].

A reduction or complete suspension of basal insulin [8], reduction of prandial insulin [9], increase in carbohydrate consumption [10] or combinations of these strategies [11] are recommended to prevent exercise-induced hypoglycaemia. Any adjustments must be guided by factors such as the type of exercise (e.g. aerobic, resistance or interval), its intensity, duration and time relative to meals; all of which modulate glucose variation. Despite these recommendations, exerciseinduced hypoglycaemia remains common [12]. The development of novel strategies to control glucose excursions during exercise remains a major unmet need.

Recently, interest in the external artificial pancreas as a promising treatment option for patients with type 1 diabetes has gained momentum [13]. In this system, subcutaneous glucose sensor readings are continuously transmitted to a mathematical dosing algorithm which dynamically controls the delivery rates of hormonal infusion pumps [13]. Two configurations of the artificial pancreas, single hormone (insulin only) and dual hormone (insulin and glucagon), have been shown to offer tighter glucose control compared with conventional pump therapy [14-17]. However, although the addition of glucagon has the potential to further lower hypoglycaemia, this comes at the expense of increased cost and device complexity [18-20].

Several approaches have been investigated for the management of exercise during the operation of the single-hormone artificial pancreas [18-22]. A fully reactive system that is driven by glucose sensor data alone fails to prevent exerciseinduced hypoglycaemia, particularly because the delay in insulin absorption reduces the effectiveness of its suspension after the start of exercise [21]. The addition of a heart rate or activity sensor to the single-hormone artificial pancreas might reduce the risk of hypoglycaemia, although at the expense of an increased burden for the patient (via wearing multiple sensors) [22]. The use of glucagon in the dual-hormone artificial pancreas might be particularly beneficial during exercise, but limited data exist to quantify the additional benefits compared with the single-hormone artificial pancreas. One study compared the two systems over $24 \mathrm{~h}$ that included physical activity, but was not sufficiently powered to detect differences related to exercise [18]. Moreover, the exercise session in this study was performed $2.5 \mathrm{~h}$ after a meal, was preceded by a snack and was not announced to the artificial pancreas.

In this study, we conducted the first randomised crossover trial to compare the efficacy of single- and dual-hormone artificial pancreases in preventing exercise-induced hypoglycaemia in adults with type 1 diabetes. Two types of exercise sessions were included: continuous and interval. These sessions were not preceded by carbohydrate ingestion but were announced to the algorithm 20 min prior to their commencement.

\section{Methods}

Study design and participants An open-label, randomised, crossover study was performed to compare the efficacy of the single-hormone vs the dual-hormone artificial pancreas during continuous exercise and interval exercise in adults with type 1 diabetes. Each participant underwent four interventions. Adults aged 18 years or older who had a clinical diagnosis of type 1 diabetes for at least 1 year, had been on insulin pump therapy for at least 3 months and whose $\mathrm{HbA}_{1 \mathrm{c}}$ levels were $\leq 12 \%(\leq 107.7 \mathrm{mmol} / \mathrm{mol})$ were invited to participate. Exclusion criteria included clinically significant microvascular complications, any acute macrovascular event in the past 3 months, abnormal blood panel findings and/or anaemia, and pregnancy. Participants with a severe hypoglycaemic episode within 2 weeks of screening or another serious medical illness likely to interfere with their ability to complete the exercise sessions were also excluded. Recruitment was carried out at the diabetes clinic of the Institut de recherches cliniques de Montréal (IRCM), QC, Canada. The study was approved by the IRCM Ethics Committee and conducted according to the 
declaration of Helsinki. All participants provided written informed consent.

Participants were first randomised to the type of exercise and then to the type of artificial pancreas (single or dual hormone). Blocked randomisation (size of four) with a 1:1:1:1 ratio was used to generate allocation sequences for the interventions. One of the study coordinators carried out the randomisation and placed the results in a sealed envelope that was open at the end of the admission visit. Participants were blinded to the sensor glucose readings and to the hormone infusion rates throughout the intervention visits.

Procedures During the admission visit, medical data, $\mathrm{HbA}_{1 \mathrm{c}}$ level and anthropometric measures were obtained. Records of insulin therapy over the past 3 days were collected, including the total daily insulin dose, basal rates and carbohydrate/ insulin ratios. Physical fitness was assessed using a protocol adapted from Storer et al [23]: participants underwent a graded exercise test on an ergocycle (Ergoline 900, Bitz, Germany) and the highest $30 \mathrm{~s}$ average oxygen uptake value obtained represented the $\dot{V} \mathrm{O}_{2 \text { peak }}$.

Continuous exercise consisted of a $60 \mathrm{~min}$ session at $60 \%$ $\dot{V} \mathrm{O}_{2 \text { peak }}$, and the interval exercise consisted of 2 min alternating periods of $85 \%$ and $50 \% \dot{V} \mathrm{O}_{2 \text { peak }}$ for $40 \mathrm{~min}$, with two 10 min periods at $45 \% \dot{V} \mathrm{O}_{2 \text { peak }}$ at the beginning and at the end of the exercise session. Both exercises were matched for energy expenditure. Participants were told to avoid moderate to high intensity exercise during the day preceding and the day of the intervention.

A glucose sensor (Dexcom G4 Platinum, Dexcom, San Diego, CA, USA) was inserted at least $24 \mathrm{~h}$ before each intervention and calibrated according to the manufacturer's recommendation by patients using their own glucose meters. The use of paracetamol was prohibited during the study to avoid potential enzymatic interference with the sensor measurements [24].

Participants consumed a standardised lunch (50 g carbohydrate for women, $70 \mathrm{~g}$ carbohydrate for men) around noon on the intervention day. An accelerometer (SenseWear Armband MF-SW, Bodymedia, Pittsburgh, PA, USA) was used to assess energy expenditure. Artificial pancreas control started at 15:30 hours, a carbohydrate-free snack was given at 15:45 hours, and exercise was announced to the algorithm at 17:40 hours, started at 18:00 hours and followed by a $30 \mathrm{~min}$ recovery period, ending at 19:30 hours. The artificial pancreas was then disconnected, a standardised dinner (60 g carbohydrate for women, $80 \mathrm{~g}$ carbohydrate for men) was served to participants and they were then discharged. Participants were asked to keep their glucose sensor on until the next morning to monitor post-exercise overnight glucose control.

Insulin aspart (B28Asp human insulin; Novorapid, Novo Nordisk Mississauga, ON, Canada) and glucagon (Eli Lilly Canada, Toronto, ON, Canada) were administered subcutaneously via infusion pumps (MiniMed Paradigm Veo, Medtronic, Northridge, CA, USA). Glucagon was reconstituted at the start of the dual-hormone artificial pancreas visit and was given as microboluses. The staff manually entered real-time sensor readings every $10 \mathrm{~min}$ into the dosing algorithm that was running on a laptop computer. Recommendations of hormonal delivery (insulin and glucagon) were then generated by the algorithm and applied manually by staff through the infusion pumps. Recommendations of the dosing algorithm were adhered to irrespective of the performance of the glucose sensor. The glucose sensors were neither recalibrated nor replaced in the event of inaccuracy.

Venous blood samples were withdrawn every $30 \mathrm{~min}$ from 15:30 to $18: 00$ hours, every $10 \mathrm{~min}$ from 18:00 to 19:00 hours (during exercise) and every 15 min from 19:00 to 19:30 hours (recovery period). Blood was directly processed to measure plasma glucose levels using a YSI2300 STAT Plus analyser (Yellow Springs, OH, USA) and samples were stored for subsequent measurement (in duplicate) of insulin and glucagon levels using an immunoassay (Millipore, Billerica, MA, USA). For hypoglycaemia treatment, if plasma glucose was $<3.3 \mathrm{mmol} / 1$ with symptoms or $<3.0 \mathrm{mmol} / 1$ irrespective of symptoms, $20 \%$ dextrose ( $7.5 \mathrm{~g}$ carbohydrate) was i.v. infused. Repeated dextrose infusions were administered until glucose level rose above $4.0 \mathrm{mmol} / \mathrm{l}$. The i.v. glucose route was used because expired gas samples were analysed throughout the test using a facial mask.

The algorithm for the artificial pancreas was initiated using the participant's weight, the time and carbohydrate amount of the last meal, and the basal and total insulin daily doses [18-20]. Insulin delivery was based on a proprietary dosing algorithm using a model predictive control. Glucagon delivery followed logical rules that considered glucose concentration estimates and their trends. Insulin delivery algorithms were identical in both the single- and dual-hormone artificial pancreas systems, except that the insulin delivery algorithm took account of the glucagon taken onboard via the dual-hormone artificial pancreas [18-20]. The target glucose level was set at $5.3 \mathrm{mmol} / \mathrm{l}$. Upon the announcement of exercise, the target glucose level was re-set to $8.3 \mathrm{mmol} / \mathrm{l}$. The original target level of $5.3 \mathrm{mmol} / \mathrm{l}$ was resumed directly at completion of the $60 \mathrm{~min}$ exercise session.

Outcomes Outcomes were recorded and analysed over a $90 \mathrm{~min}$ period (60 min of exercise and $30 \mathrm{~min}$ of recovery). The primary outcome was the number of participants experiencing exercise-induced hypoglycaemia requiring treatment. Secondary outcomes included the number of participants with plasma glucose below $3.9 \mathrm{mmol} / \mathrm{l}$, a decrease in glucose levels, a decremental AUC from the start of exercise, the AUC for plasma glucose levels below $4.0 \mathrm{mmol} / \mathrm{l}$, the percentage of time spent with plasma glucose levels below $4.0 \mathrm{mmol} / \mathrm{l}$ and the percentage of time in which plasma glucose levels were within the target range $(4.0-10.0 \mathrm{mmol} / \mathrm{l})$. 
Statistical analysis We anticipated that $70 \%$ of participants would experience hypoglycaemia with the single-hormone but not with the dual-hormone artificial pancreas, that $5 \%$ would have hypoglycaemia with the dual-hormone but not with the single-hormone artificial pancreas, while the remaining $25 \%$ of patients would have hypoglycaemia with both or neither. After correcting for multiple comparisons $(\alpha=0.05 / 2)$, 14 participants were needed to achieve $80 \%$ statistical power (using power calculations for the McNemar test). To account for dropout, 20 participants were recruited for the study.

Data collected separately for each type of exercise and pooled data for both exercise types were analysed. A McNemar test was used to compare the number of participants with at least one hypoglycaemic event requiring treatment during an intervention (i.e. hypoglycaemia frequency) and to compare participants who experienced glucose levels lower than $3.9 \mathrm{mmol} / \mathrm{l}$. For all continuous outcomes, a multivariate linear mixed effect model with the treatment sequence (fixed effect), individual nested within sequence (random effect), period (fixed effect) and treatment (fixed effect) entered as covariates was applied. For analysis of overnight data, the same model was used to compare the percentage of time spent in hypoglycaemia, at the target glucose level and in hyperglycaemia according to glucose sensor readings, with hypoglycaemia treatment during exercise added as a covariate. One-way ANOVA was used to compare energy expenditure among the four types of interventions. A threshold of 5\% was used to set statistical significance. As pre-planned, modified intention-to-treat analyses were performed: patients who completed at least two visits were included in the analysis. R software version 3.1.2 (https://cran.rproject.org/bin/windows/base/old/3.1.2/) was used for the analysis and data are presented as numbers, percentages, means $\pm \mathrm{SD}$ and/or medians (interquartile range $[\mathrm{IQR}]$ ).

\section{Results}

Between June 2014 and April 2015, 17 participants (eight women) were enrolled in the study, with a mean age of $37.2 \pm 13.6$ years, type 1 diabetes duration of $23.1 \pm 11.7$ years, $\mathrm{HbA}_{1 \mathrm{c}}$ level of $8.0 \pm 1.0 \%$ [63.9 $\left.\pm 10.2 \mathrm{mmol} / \mathrm{mol}\right]$ ), insulin dose of $46.2 \pm 15.8 \mathrm{U} /$ day and BMI of $25 \pm 4.0 \mathrm{~kg} / \mathrm{m}^{2}$. Participants had different levels of physical fitness: mean $\dot{V} \mathrm{O}_{\text {2peak }} 34.2 \pm 5.4$ (minimum 19.9, maximum 42.6) $\mathrm{ml} \mathrm{kg}^{-1}$ $\min ^{-1}$ (electronic supplementary material [ESM] Table 1). Twenty participants were initially enrolled, of whom two dropped out after the admission visit, one dropped out after completing only one intervention and two dropped out after completing two to three interventions (reasons for dropping out are detailed in Fig. 1).

Figures 2 and 3 show plasma glucose and glucagon levels, insulin and glucagon delivery rates, and hypoglycaemic events at all intervention visits. Mean glucose levels at the start of exercise were between 6.3 and $7.1 \mathrm{mmol} / \mathrm{l}$ for all study conditions, with no significant difference between glucose levels (Table 1). The mean \pm SD estimated energy expenditure was similar at all four visits: single-hormone system during continuous exercise, $1585.7 \pm 489.1 \mathrm{~kJ}$; dual-hormone system during continuous exercise, $1651.4 \pm 401.7 \mathrm{~kJ}$; singlehormone system during interval exercise, $1640.1 \pm 380.7 \mathrm{~kJ}$; dual-hormone during interval exercise, $1627,6 \pm 493.7 \mathrm{~kJ}$ $(p=0.98)$.

During continuous exercise, four participants (23.5\%) experienced at least one hypoglycaemic event requiring treatment (plasma glucose $<3.3 \mathrm{mmol} / 1$ with symptoms or $<3.0 \mathrm{mmol} / 1$ irrespective of symptoms) with the singlehormone artificial pancreas, compared with two participants $(11.8 \%)$ with the dual-hormone artificial pancreas $(p=0.5)$. During interval exercise, six participants (40.0\%) experienced at least one hypoglycaemic event requiring treatment with the single-hormone artificial pancreas compared with one participant $(6.25 \%)$ with the dual-hormone artificial pancreas $(p=0.07$; Table 1). In total, there were 15 events with the single-hormone artificial pancreas (seven during continuous and eight during interval exercise). With the dual-hormone artificial pancreas, the number of events was reduced to three (two during continuous and one during interval exercise; Table 1). The glucose sensor over-read by a mean $\pm \mathrm{SD}$ of $1.1 \pm 0.8 \mathrm{mmol} / 1$ for the 15 hypoglycaemic events under single-hormone control compared with $3.1 \pm 2.1 \mathrm{mmol} / \mathrm{l}$ under dual-hormone control $(5.5,2.2$ and $1.6 \mathrm{mmol} / \mathrm{l}$ for the three individual events). At the $3.9 \mathrm{mmol} / \mathrm{l}$ threshold during continuous exercise, nine participants $(52.9 \%)$ had hypoglycaemia with the single-hormone artificial pancreas compared with three $(17.6 \%)$ with the dual-hormone artificial pancreas $(p=0.07)$. Similarly, during interval exercise, seven participants $(46.7 \%)$ had hypoglycaemia with the single-hormone artificial pancreas compared with one $(6.25 \%)$ with the dualhormone artificial pancreas $(p=0.04)$.

The median time spent with plasma glucose levels below $4.0 \mathrm{mmol} / 1$ was significantly higher with the single-hormone artificial pancreas than with the dual-hormone artificial pancreas: $22.5 \%$ (IQR $0-48.3)$ vs $0 \%(0-0 \% ; p=0.006)$ during continuous exercise and $0 \%(0-30.4 \%)$ vs $0 \%(0-0 \%$; $p=0.03)$ during interval exercise. Similarly, the median AUC for hypoglycaemia $(<4.0 \mathrm{mmol} / \mathrm{l})$ was higher for the single-hormone system compared with the dual-hormone system: $63.3(0-154.6)$ vs $0(0-0) \mathrm{mmol} / \mathrm{l} \times \min (p=0.008)$ for continuous and $0(0-89.9)$ vs $0(0-0) \mathrm{mmol} / 1 \times \min (p=0.02)$ for interval exercise (Table 1). With the single-hormone system, participants also spent a lower median percentage of time at target glucose levels $(4.0-10.0 \mathrm{mmol} / \mathrm{l}): 68.1 \%$ (51.6$100 \%)$ vs $100 \%(100-100 \% ; p=0.004)$ during continuous exercise and $72.5 \%(60.4-100 \%)$ vs $100 \%(100-100 \%$; $p=0.11$ ) during interval exercise (Table 1). 
Fig. 1 Trial flow chart. SAP, single-hormone artificial pancreas; DAP, dual-hormone artificial pancreas

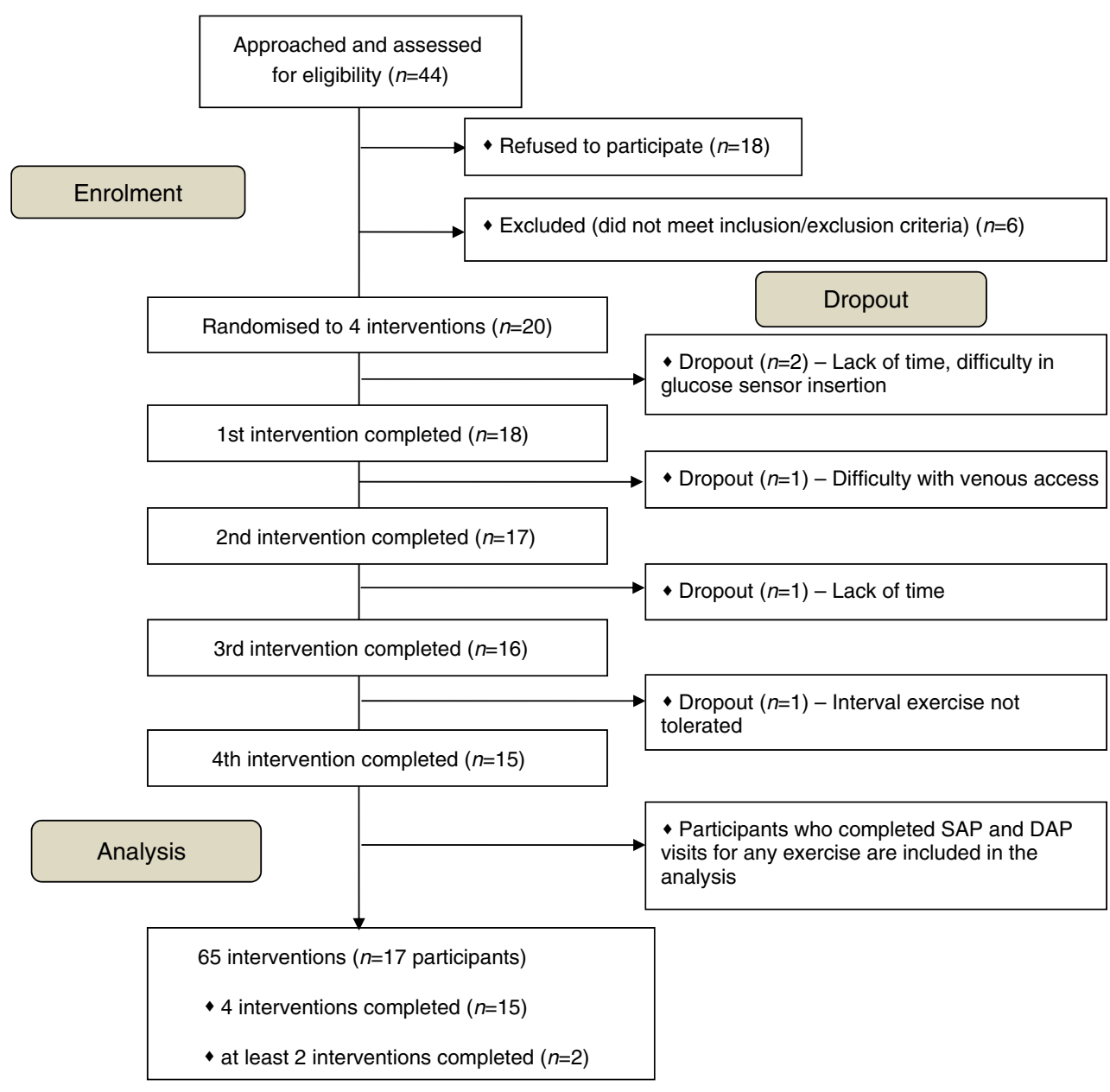

From the time exercise was announced until the end of the recovery period, the insulin infusion rate did not significantly differ between the single-hormone and dual-hormone systems: the means were $0.30 \pm 0.33 \mathrm{U} / \mathrm{h}$ and $0.40 \pm 0.36 \mathrm{U} / \mathrm{h}$ $(p=0.7)$, respectively, during continuous exercise and $0.32 \pm 0.35 \mathrm{U} / \mathrm{h}$ and $0.44 \pm 0.3 \mathrm{U} / \mathrm{h}(p=0.2)$, respectively, during interval exercise. The plasma insulin concentrations did not significantly differ between the two systems during both exercise types (Table 1). With the dual-hormone artificial pancreas, more glucagon was needed during continuous $(0.126 \pm 0.057 \mathrm{mg})$ than during interval $(0.093 \pm 0.068 \mathrm{mg})$ exercise $(p=0.03)$. However, the mean plasma glucagon concentrations did not significantly differ between the two types of exercise: continuous exercise, $210.6 \pm 71.4 \mathrm{ng} / \mathrm{l}$, interval exercise, $210.8 \pm 96.0 \mathrm{ng} / \mathrm{l}(p=0.97)$. These correspond to an increase by a factor of 2.6 and 2.7 , respectively, compared with single-hormone artificial pancreas visits. No participant reported side-effects related to glucagon administration.

The pooled data comparing the two systems regardless of exercise type followed the same trends seen with the separate analyses. Glucagon use in dual-hormone artificial pancreas further prevented exercise-induced hypoglycaemia compared with the single-hormone artificial pancreas; the percentages of participants with hypoglycaemia requiring treatment and plasma glucose $<3.9 \mathrm{mmol} / \mathrm{l}$ were reduced by $22 \%(p=0.02)$ and $38 \%$ ( $p=0.003)$, respectively, with the dual-hormone system (ESM Table 2). Similarly, the median time spent in hypoglycaemia (plasma glucose $<4.0 \mathrm{mmol} / \mathrm{l}$ ) was decreased by $11 \% \quad(p=0.0001)$ while that spent at target levels (plasma glucose 4.0-10.0 mmol/1) was increased by $28.6 \%(p=0.003)$ as an added benefit of glucagon use. Details about the pooled analysis are provided in Fig. 4 and ESM Table 2.

The analysis of time spent at target glucose, in hypoglycaemic and in hyperglycaemic ranges according to overnight glucose sensor readings (conventional pump therapy was applied during the night) is detailed in ESM Table 3. After single- and dual-hormone system artificial pancreas interventions, the mean overnight time spent in the target range was $54.4 \pm 26.3 \%$ vs $70.5 \pm 21.6 \%(p=0.13)$ for continuous exercise, and $52.2 \pm 31.7 \%$ vs $77.4 \pm 15.7 \%$ for interval exercise $(p=0.03)$. There was no difference in the time spent in hypoglycaemia after single- vs dual-hormone interventions. Lower mean glucose levels were seen in the nights that followed dual-hormone interventions $8.1 \pm 2.1 \mathrm{mmol} / \mathrm{l}$ for continuous and $7.2 \pm 1.6 \mathrm{mmol} / \mathrm{l}$ for interval than the nights following 


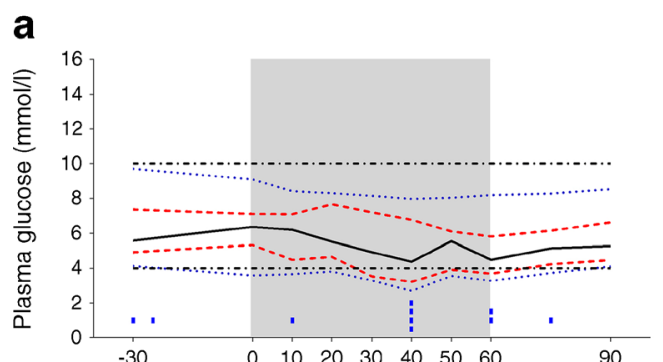

b

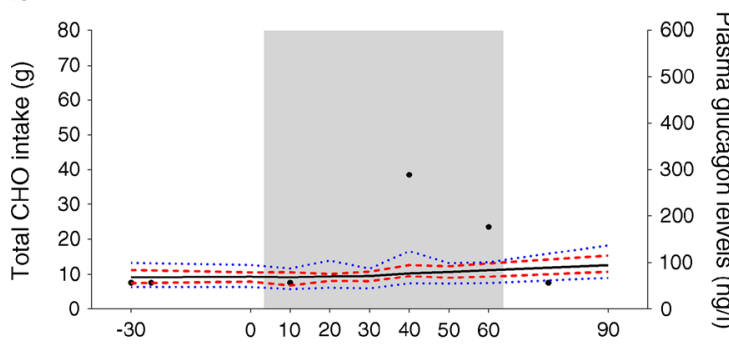

C

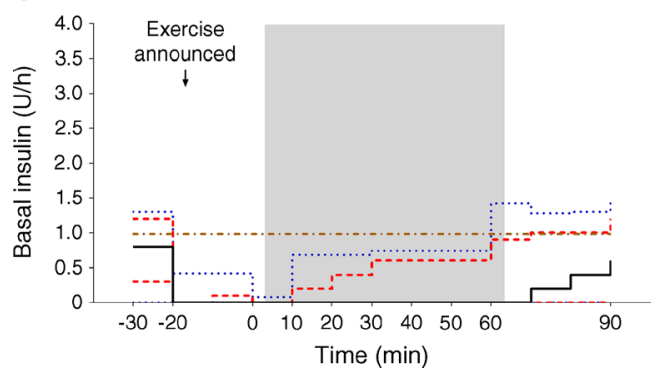

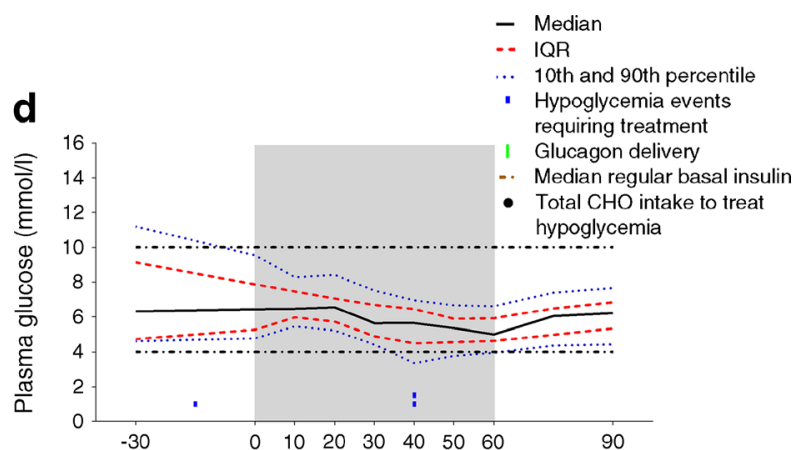

e

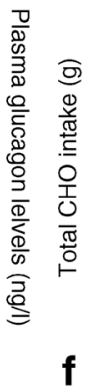

f

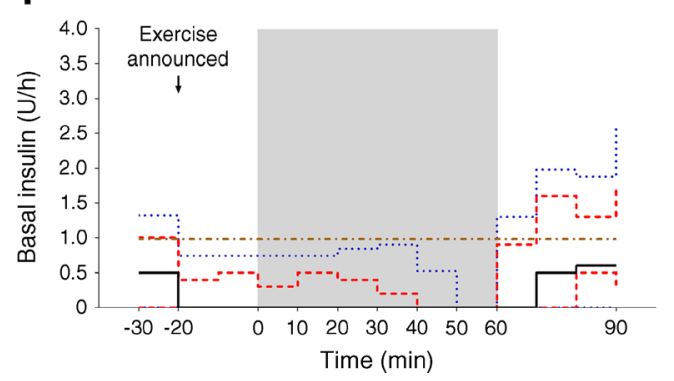

Fig. 2 Continuous exercise graphs for participants with $(\mathbf{a}-\mathbf{c})$ single-hormone artificial pancreases and $(\mathbf{d}-\mathbf{f})$ dual-hormone artificial pancreases. Shaded area corresponds to the exercise session. $\mathrm{CHO}$, carbohydrate

single-hormone interventions $9.1 \pm 2.0 \mathrm{mmol} / \mathrm{l}$ and 9.4 $\pm 2.8 \mathrm{mmol} / \mathrm{l}$ for continuous and interval exercise, respectively.

\section{Discussion}

The artificial pancreas has the potential to revolutionise diabetes treatment [13]. The dual-hormone version in particular is expected to improve glucose control during situations with an increased risk of hypoglycaemia, such as exercise [25]. We report the first clinical trial designed to directly compare the singlehormone and dual-hormone artificial pancreas systems during two different types of exercise in adults with type 1 diabetes. Our results suggest that adding glucagon to the artificial pancreas may reduce exercise-induced hypoglycaemia and improve glucose control during both continuous and interval exercise.

Previous studies have compared conventional insulin pump therapy to either a single-hormone or dual-hormone artificial pancreas during exercise $[15,26]$. Only one study directly reported exercise-related outcomes when comparing the two artificial pancreas systems (conventional vs single-hormone vs dual- hormone) [18]. This study included a continuous type of exercise which was postprandial and preceded by a carbohydrate rich snack. Hypoglycaemic events were rare with both systems (7\% and $14 \%$ with single- and dual-hormone systems, respectively) compared with a conventional insulin pump (38\%) [18]. In the present study, in which exercise was performed prior to dinner without carbohydrate-containing snack consumption, the addition of glucagon resulted in a reduction of hypoglycaemic events and of time spent in the hypoglycaemic range. All three hypoglycaemic events that happened under dual-hormone control were probably due to sensor over-reading rather than to algorithm recommendations. This type of event is expected to be decreased or eliminated in the future with the use of glucose sensors with better performance. However, glucose sensor performance is reduced during exercise, leading to an overall overestimation of glucose values compared with rest: in our study the mean absolute relative difference (MARD) for Dexcom measurements of plasma glucose was $13.8 \pm 12.0 \%$ at rest, which increased to $22.5 \pm 17.2 \%$ during exercise [27]. A similar MARD difference between rest and exercise has been observed with other sensors (e.g. Enlite Veo) [27]. 
a

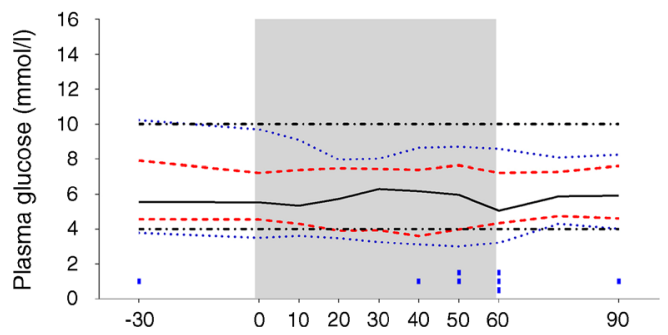

b

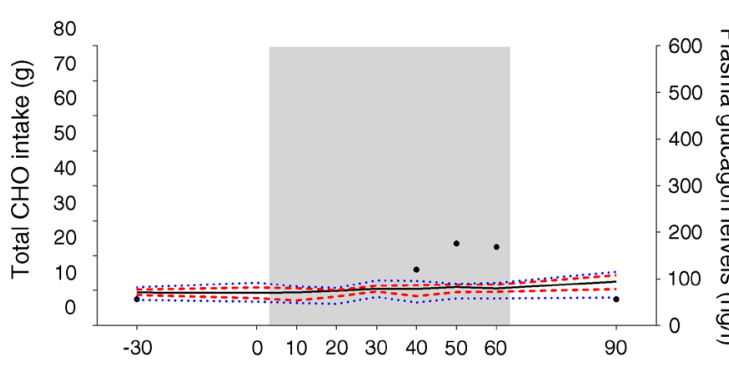

C

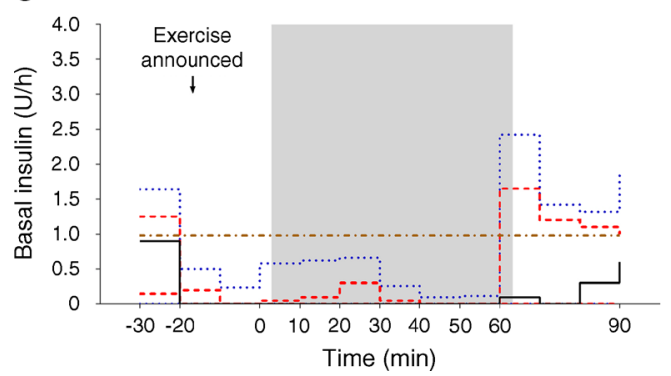

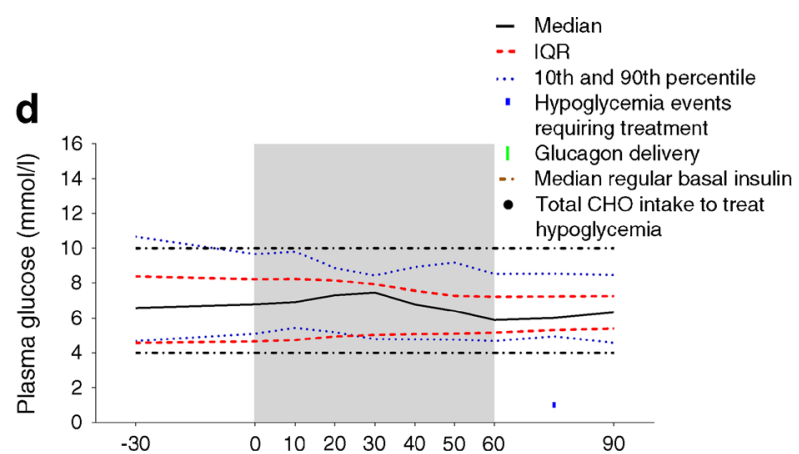

e

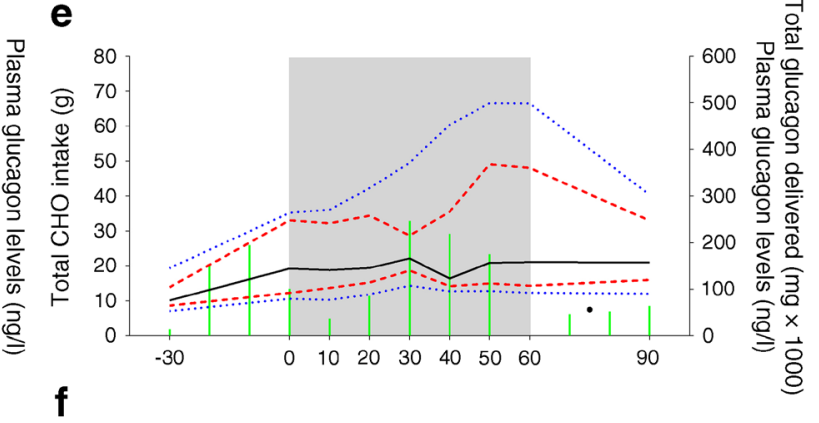

f

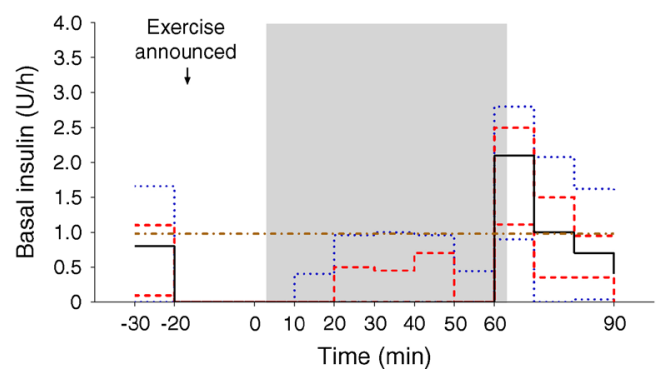

Fig. 3 Interval exercise graphs for participants with $(\mathbf{a}-\mathbf{c})$ single-hormone artificial pancreases and $(\mathbf{d}-\mathbf{f})$ dual-hormone artificial pancreases. Shaded area corresponds to the exercise session

Following dual-hormone interventions, a more favourable overnight glycaemic profile was also noted compared with single-hormone interventions. This was reflected by a higher percentage of time spent at target and at lower mean glucose levels after the dual-hormone interventions. This may be due to the more frequent carbohydrate consumption that was needed during the single-hormone visits. However, interpretation of this data is limited by the fact that this part of the protocol was unsupervised, and there is therefore a lack of information about pre-dinner insulin boluses, overnight hypoglycaemic events and snack consumption.

Management of glucose fluctuations during and following physical activity is influenced by several factors such as the timing, duration and intensity of exercise, as well as the participant's physical fitness and individual insulin treatment regimen $[6,28]$. The strategies proposed to avoid exerciseinduced hypoglycaemia, although helpful, are not sufficient $[8,29]$. Patients with type 1 diabetes are challenged by the need to plan their physical activity well ahead to either adjust their insulin injections or pump infusion rates and prandial boluses. Despite early planning, they are often compelled to consume additional carbohydrates, which can lead to excess caloric intake and may increase the risk of subsequent hyperglycaemia [29]. Patients with type 1 diabetes thus face many barriers and rarely meet the recommended physical activity levels, despite the proven health and cardiovascular benefits of exercise [2,3]. In this trial, exercise was not preceded by carbohydrate consumption but was announced to the artificial pancreas algorithm $20 \mathrm{~min}$ ahead of the session, which is a practical timing for patients. The addition of glucagon not only decreased the risk of hypoglycaemia but also improved the total time spent at target plasma glucose levels. Accordingly, it would be essential to evaluate the impact of the artificial pancreas (more specifically, the dual-hormone version) on modulating the behaviour and attitude of patients with type 1 diabetes towards exercise. Whether this would translate into more active lifestyles, improved glycaemic control and cardiovascular metabolic profile is important to assess in future long-term outpatient studies.

The amounts of insulin delivered were comparable between the two artificial pancreas systems. Therefore, the improvement in glucose control seen with the dual-hormonal 


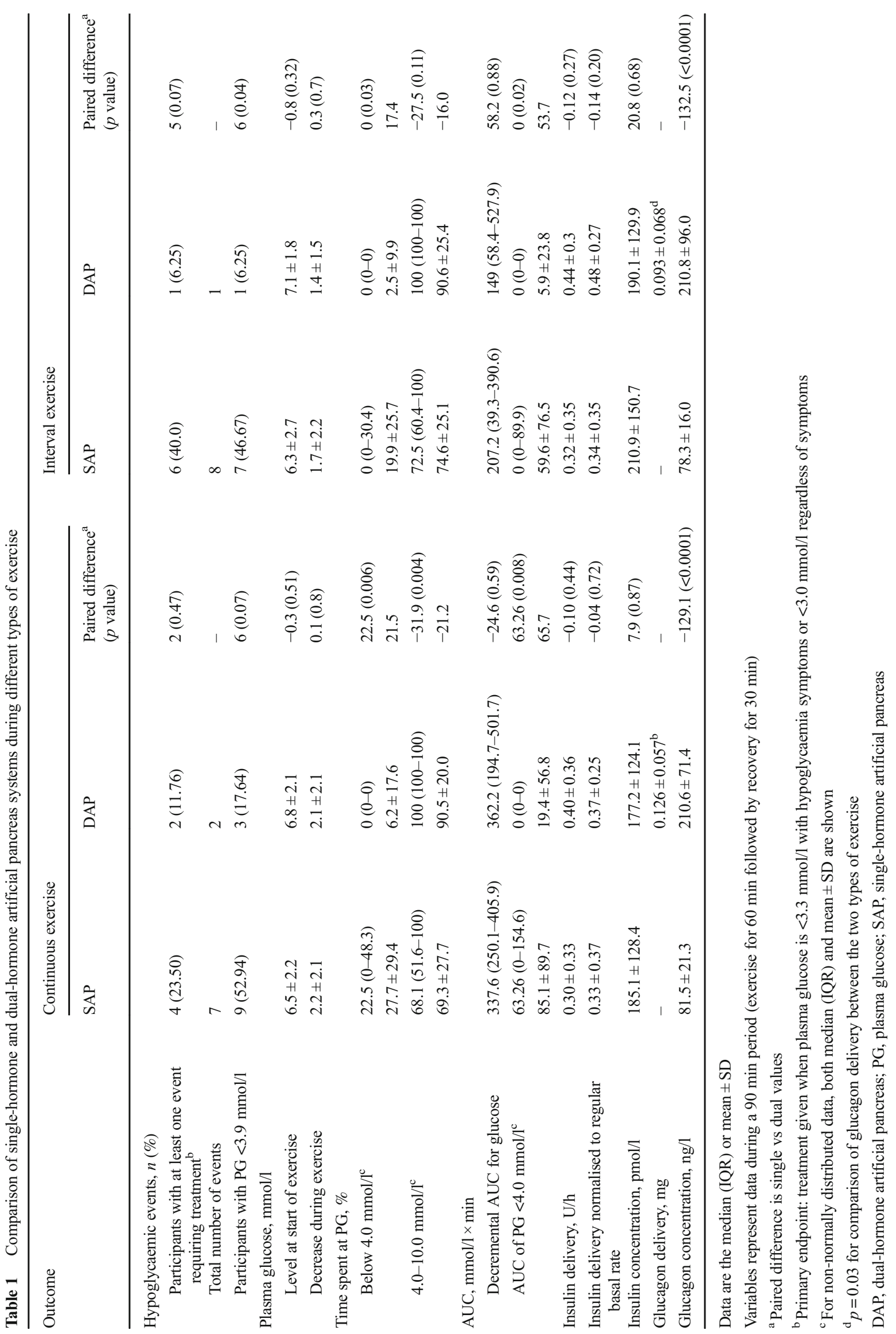


Fig. 4 Main results of the pooled analysis. (a) Percentage of participants with hypoglycaemia necessitating treatment, (b) percentage of participants with $\mathrm{PG}<3.9 \mathrm{mmol} / \mathrm{l}$, (c) percentage of time spent at $\mathrm{PG}<4 \mathrm{mmol} / \mathrm{l}$, (d) Percentage of time spent in target (PG 4-10 mmol/l). For (c) and (d), data are presented as means \pm SD. ${ }^{\dagger} p=0.02,{ }^{\dagger \dagger} p=0.003$, ${ }^{\dagger \dagger} p=0.0001$. DAP, dualhormone artificial pancreas; $\mathrm{PG}$, plasma glucose; SAP, singlehormone artificial pancreas a
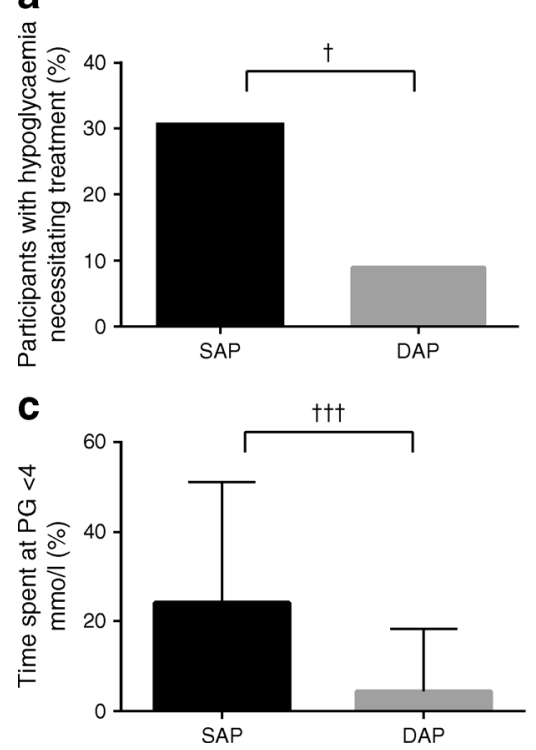

b

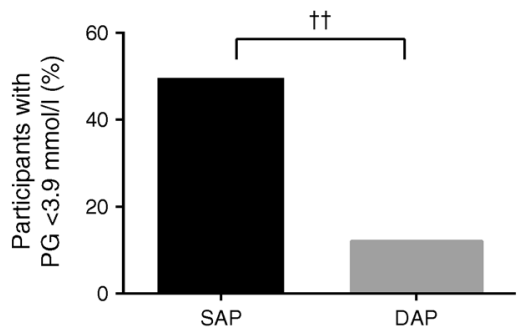

d

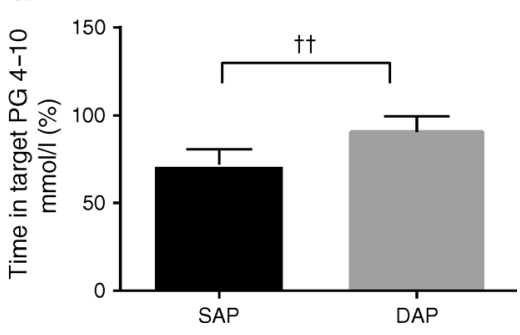

artificial pancreas system is solely attributable to the addition of glucagon. Figures 2 and 3 show that more carbohydrate consumption was needed during glucose control by the single-hormone artificial pancreas, whereas this was largely replaced with glucagon during control by the dual-hormone artificial pancreas. Of note, plasma glucagon levels were not increased in response to exercise during the single-hormone artificial pancreas visits (Figs 2 and 3), while glucagon administration by the dual-hormone system increased the plasma glucagon concentration by a factor of 2.6 compared with use of the single-hormone artificial pancreas. Glucagon levels had been reported to increase by around twofold in healthy individuals performing continuous exercise at $50 \% \dot{V} \mathrm{O}_{2 \text { peak }}$ for $75 \mathrm{~min}$ and by 1.4 fold during continuous cycling at $40 \% \dot{V} \mathrm{O}_{2 \text { peak }}$ for $60 \mathrm{~min}[30,31]$. Thus, the algorithm of the dual-hormone artificial pancreas could prevent hypoglycaemia by raising glucagon concentrations to the levels observed in healthy exercising individuals.

For this trial, continuous and interval types of exercise were chosen because they confer different glycaemic variability and hypoglycaemic risks in patients with diabetes [32, 33]. In our study, $69 \%$ more glucagon was needed during continuous exercise compared with the interval exercise sessions. This is in accordance with the lower rates of glucose decline reported during intermittent compared with continuous exercise in type 1 diabetes [33] and has been attributed to enhanced endogenous glucose production and attenuated utilisation of glucose during intermittent exercise [34].

Our study had some limitations. It was conducted in a controlled inpatient environment; therefore, future trials in real-life settings will be needed to confirm our results. Different scenarios such as pre-exercise carbohydrate ingestion, early postprandial, longer or higher intensity exercise, as well as unannounced exercise will need to be tested to further evaluate the effects of glucagon addition to the artificial pancreas. For the overnight sensor readings analysis, detailed information about food intake and hypoglycaemia correction after discharge was not collected, thus limiting the interpretation of these results. A manually controlled artificial pancreas system was used, necessitating future testing in an automated system, but our approach bypasses potential problems related to connectivity. Blinding of patients to the type of intervention was difficult in this study but is unlikely to alter the results.

In this study, the dual-hormone artificial pancreas reduced the risk of exercise-induced hypoglycaemia compared with the single-hormone artificial pancreas during two types of exercise in adults with type 1 diabetes. This is the first trial to quantify the added benefit of glucagon use in the artificial pancreas during exercise. Larger studies in which exercise is performed in free-living conditions are justified to confirm these results and identify the situations or patients who would most benefit from glucagon addition.

Acknowledgements We are thankful to all the participants who dedicated their time and effort to complete this study. We would like to equally acknowledge the contributions of the nurses at the Institut de recherches cliniques de Montréal (Montréal, QC, Canada), A. Magnan, M. Dallaire and M. Forde Lewis. Some of the data were presented as abstracts at the 75th Scientific Sessions of the ADA, Boston, MA, 5-9 June 2015, and the 9th International Conference on Advanced Technologies And Treatments For Diabetes, Milan, Italy, 3-6 February 2016.

Funding This study was supported by funds from the Société Francophone du Diabète, Diabète Québec, a Fondation J-A de Sève chair held by RR-L, a Best and Banting Canadian Institutes of Health Research 
(CIHR) fellowship held by AH, graduate CIHR, Fonds de recherche du Québec - Santé (FRQS) and Eli Lilly scholarship grants held by NT, a graduate Natural Sciences and Engineering Research Council of Canada award held by AE, and a senior FRQS scholar award held by RR-L. Glucagon and insulin were in-kind contributions from Eli Lilly Canada and Novo Nordisk, respectively.

Duality of interest statement $\mathrm{AH}$ has received consultant's or speaker's honorariums from SNELL Medical Communication and the Diabetic Children's Foundation. RR-L has received consultant's or speaker's honorariums or grants from AstraZeneca, Becton Dickinson, Boehringer Ingelheim, Eli Lilly, Janssen, Lifescan, Medtronic, Merck, Novartis, Neomed, Novo Nordisk, Roche, Sanofi-Aventis, Takeda, and Valeant. AH and RR-L submitted a report of invention of the dosing algorithm to their institution. LL received advisory fees from Eli Lilly Canada, non-financial support from Eli Lilly Canada, research support from Novo Nordisk and research support from Merck Canada. LL, VM, $\mathrm{AH}$ and RR-L own intellectual property in the area of artificial pancreas. All other authors report that there is no duality of interest associated with their contribution to this manuscript.

Contribution statement NT and CS coordinated the study; AH, RR-L, LL, J-LC and VM participated in the design of the study; AH designed and implemented the dosing algorithm; NT, AE and CS carried out data acquisition; NT, AE and VM carried out data processing and prepared graphs; NT, $\mathrm{ML}$ and $\mathrm{AH}$ carried out the statistical analysis and data interpretation; NT drafted the manuscript, which was revised critically and edited by all the coauthors; and all authors approved the final version of the manuscript. NT, AH and RR-L had full access to the data and take responsibility for the integrity of the data and the accuracy of the data analysis.

\section{References}

1. Armstrong MJ, Sigal RJ (2013) Physical activity clinical practice guidelines: what's new in 2013? Can J Diabetes 37:363-6

2. Chimen M, Kennedy A, Nirantharakumar K, Pang TT, Andrews R, Narendran P (2012) What are the health benefits of physical activity in type 1 diabetes mellitus? A literature review. Diabetologia 55: $542-51$

3. Tielemans SM, Soedamah-Muthu SS, de Neve M et al (2013) Association of physical activity with all-cause mortality and incident and prevalent cardiovascular disease among patients with type 1 diabetes: The EURODIAB Prospective Complications Study. Diabetologia 56:82-91

4. Plotnikoff RC, Taylor LM, Wilson PM et al (2006) Factors associated with physical activity in Canadian adults with diabetes. Med Sci Sports Exerc 38:1526-34

5. Brazeau A-S, Rabasa-Lhoret R, Irene S, Mircescu H (2008) Barriers to physical activity among patients with type 1 diabetes. Diabetes Care 31:2108-9

6. McMahon SK, Ferreira LD, Ratnam N et al (2007) Glucose requirements to maintain euglycemia after moderate-intensity afternoon exercise in adolescents with type 1 diabetes are increased in a biphasic manner. J Clin Endocrinol Metab 92:963-8

7. Tansey MJ, Tsalikian E, Beck RW et al (2006) The effects of aerobic exercise on glucose and counterregulatory hormone concentrations in children with type 1 diabetes. Diabetes Care 29:20-5

8. Tsalikian E, Kollman C, Tamborlane WB et al (2006) Prevention of hypoglycemia during exercise in children with type 1 diabetes by suspending basal insulin. Diabetes Care 29:2200-4
9. Rabasa-Lhoret R, Bourque J, Ducros F, Chiasson JL (2001) Guidelines for premeal insulin dose reduction for postprandial exercise of different intensities and durations in type 1 diabetic subjects treated intensively with a basal-bolus insulin regimen (ultralente-lispro). Diabetes Care 24:625-30

10. Riddell MC, Bar-Or O, Ayub BV, Calvert RE, Heigenhauser GJ (1999) Glucose ingestion matched with total carbohydrate utilization attenuates hypoglycemia during exercise in adolescents with IDDM. Int J Sport Nutr 9:24-34

11. Campbell MD, Walker M, Bracken RM et al (2015) Insulin therapy and dietary adjustments to normalize glycemia and prevent nocturnal hypoglycemia after evening exercise in type 1 diabetes : a randomized controlled trial. BMJ Open Diabetes Res Care 3:e000085

12. Chu L, Hamilton J, Riddell MC (2011) Clinical management of the physically active patient with type 1 diabetes. Phys Sportsmed 39: 64-77

13. Hovorka R (2011) Closed-loop insulin delivery: from bench to clinical practice. Nat Rev Endocrinol 7:385-95

14. El-Khatib FH, Russell SJ, Magyar KL et al (2014) Autonomous and continuous adaptation of a bihormonal bionic pancreas in adults and adolescents with type 1 diabetes. J Clin Endocrinol Metab 99:1701-11

15. Thabit H, Tauschmann M, Allen JM et al (2015) Home use of an artificial beta cell in type 1 diabetes. N Engl J Med 273:2129-40

16. Haidar A, Legault L, Dallaire M et al (2013) Glucose-responsive insulin and glucagon delivery (dual-hormone artificial pancreas) in adults with type 1 diabetes: a randomized crossover controlled trial. CMAJ 185:297-305

17. Castle JR, Engle JM, El Youssef J et al (2010) Novel use of glucagon in a closed-loop system for prevention of hypoglycemia in type 1 diabetes. Diabetes Care 33:1282-7

18. Haidar A, Legault L, Messier V, Mitre TM, Leroux C, RabasaLhoret R (2015) Comparison of dual-hormone artificial pancreas, single-hormone artificial pancreas, and conventional insulin pump therapy for glycaemic control in patients with type 1 diabetes : an open-label randomised controlled crossover trial. Lancet Diabetes Endocrinol 3:17-26

19. Haidar A, Legault L, Matteau-Pelletier L et al (2015) Outpatient overnight glucose control with dual-hormone artificial pancreas, singlehormone artificial pancreas, or conventional insulin pump therapy in children and adolescents with type 1 diabetes: an open-label, randomised controlled trial. Lancet Diabetes Endocrinol 3:595-604

20. Haidar A, Rabasa-lhoret R, Legault L et al (2016) Single-and dualhormone artificial pancreas for overnight glucose control in type 1 diabetes. J Clin Endocrinol Metab 101:214-23

21. Elleri D, Allen JM, Kavita Kumareswaran K et al (2013) Closedloop basal insulin delivery over 36 hours in adolescents with type 1 diabetes randomized clinical trial. Diabetes Care 36:838-44

22. Jacobs PG, Resalat N, El Youssef J et al (2015) Incorporating an exercise detection, grading, and hormone dosing algorithm into the artificial pancreas using accelerometry and heart rate. J Diabetes Sci Technol 5:1175-84

23. Storer TW, Davis JA, Caiozzo VJ (1990) Accurate prediction of VO2max in cycle ergometry. Med Sci Sports Exerc 22:704-12

24. Maahs DM, DeSalvo D, Pyle L et al (2015) Effect of acetaminophen on CGM glucose in an outpatient setting. Diabetes Care 38:e158-e159

25. Tonoli C, Heyman E, Roelands B et al (2012) Effects of different types of acute and chronic (training) exercise on glycaemic control in type 1 diabetes mellitus: a meta-analysis. Sports Med 42:1059-80

26. Luijf YM, Devries JH, Zwinderman K et al (2013) Day and night closed-loop control in adults with type 1 diabetes. Diabetes Care 36:3882-7

27. Taleb N, Emami A, Suppere C et al (2016) Comparison of two continuous glucose monitoring systems, Dexcom G4 Platinum 
and Medtronic Paradigm Veo Enlite, at rest and during exercise. Diabetes Technol Ther 18:561-567

28. Henriksson J (1995) Influence of exercise on insulin sensitivity. J Cardiovasc Risk 2:303-9

29. Yardley JE, Sigal RJ (2015) Exercise strategies for hypoglycemia prevention in individuals with type 1 diabetes. Diabetes Spectr 28:32-8

30. Hinshaw L, Mallad A, Dalla Man C et al (2015) Glucagon sensitivity and clearance in type 1 diabetes: insights from in vivo and in silico experiments. Am J Physiol Endocrinol Metab 309:E474-E486

31. Redmon JB, Kubo SH, Robertson RP (1995) Glucose, insulin, and glucagon levels during exercise in pancreas transplant recipients. Diabetes Care 18:457-62
32. Guelfi KJ, Jones TW, Fournier PA (2005) The decline in blood glucose levels is less with intermittent high-intensity compared with moderate exercise in individuals with type 1 diabetes. Diabetes Care 28:1289-94

33. Yardley J, Mollard R, MacIntosh A et al (2013) Vigorous intensity exercise for glycemic control in patients with type 1 diabetes. Can J Diabetes 37:427-32

34. Guelfi KJ, Ratnam N, Smythe GA, Jones TW, Fournier PA (2007) Effect of intermittent high-intensity compared with continuous moderate exercise on glucose production and utilization in individuals with type 1 diabetes. Am J Physiol Endocrinol Metab 292: E865-E870 